\title{
Waist Circumference and Diabetes Risk in Colombian Population
}

\author{
Richard Buendia M.D 1,2., Monica Zambrano M.D 1,2,3, Sonia Velandia M.D.2,
} Alejandra Morales M.D. ${ }^{2}$, Adriana Alejo M.D. ${ }^{2}$, Laura Giraldo M.D. ${ }^{2}$, Diana Gamez M.D. ${ }^{2}$.

1. Pontificia Universidad Javeriana School of Medicine. 2. Colsubsidio. 3. Hospital de la Samaritana. Bogotá D.C. Colombia

Introducction.

Increased waist circumference is a cardiovascular risk factor. Currently, we have the cutoff value of International Diabetes Federation (IDF) or by LatinAmerican standards; Colombia has no studies showing the relationship between increased waist circumference and the risk of diabetes. This study aims to show the relationship between increased waist circumference by IDF criteria or LatinAmerican criteria and the risk of diabetes.

\section{Methodology.}

Cross-sectional study where multivariate analysis was performed using two models: waist circumference increased by IDF criteria ( $\geq 90 \mathrm{~cm}$ in male and $\geq 80$ $\mathrm{cm}$ in female) and waist circumference by Latin-American criteria ( $\geq 94 \mathrm{~cm}$ in male and $\geq 90 \mathrm{~cm}$ in female) and risk of diabetes; adjusted for age, sex, HDL cholesterol, and body mass index (relevant variables in univariate analysis).

Table No1 Baseline Characteristics N= 2200 patients

\begin{tabular}{|c|c|c|c|}
\hline \multirow{2}{*}{ Variable } & \multicolumn{2}{|c|}{ Diabetes } & \multirow{2}{*}{$\mathrm{p}$} \\
\hline & $\begin{array}{c}\text { NO }(n=1530 \\
(69,55 \%))\end{array}$ & $\begin{array}{c}\text { YES }(n=670 \\
(30,45 \%))\end{array}$ & \\
\hline Female $\mathrm{n}(\%)$ & $761(49,74)$ & $276(41,19)$ & $<0,001$ \\
\hline Male n (\%) & $769(50,26)$ & $394(58,81)$ & \\
\hline Age (Mean (SD)) & $65,43(12,44)$ & $66,19(12,01)$ & 0,18 \\
\hline $\begin{array}{l}\text { Weight Kgs } \\
\text { (Mean (SD)) }\end{array}$ & $71,11(12,77)$ & $74,31(13,23)$ & $<0,001$ \\
\hline $\begin{array}{l}\text { Hypertension } \\
\text { n(\%) }\end{array}$ & $1316(86,01)$ & $440(65,67)$ & $<0,001$ \\
\hline $\begin{array}{l}\text { Waist } \\
\text { Circumference } \\
\text { Increased by IDF } \\
\text { criteria } \mathrm{n}(\%)\end{array}$ & $1305(85,41)$ & $606(90,72)$ & 0,001 \\
\hline \begin{tabular}{|l} 
Waist \\
Circumference \\
Increased by \\
Latin-American \\
criteria n (\%)
\end{tabular} & $1036(67,80)$ & $512(76,65)$ & $<0,001$ \\
\hline $\begin{array}{l}\text { Body Mass Index } \\
\mathrm{Kg} / \mathrm{m} 2 \text { (Mean } \\
(\mathrm{SD}) \text { ) }\end{array}$ & $28,40(4,89)$ & $29,05(28,68)$ & \\
\hline $\begin{array}{l}\text { A1c \% (Mean } \\
\text { (SD)) }\end{array}$ & $6,03(0,51)$ & $7,74(1,72)$ & $<0,001$ \\
\hline $\begin{array}{l}\text { Creatinine } \mathrm{mg} / \mathrm{dl} \\
\text { (Mean (SD)) }\end{array}$ & $1,43(1,26)$ & $1,64(0,82)$ & 0,48 \\
\hline $\begin{array}{l}\text { Albumin Excretion } \\
\text { Rate } \mathrm{mg} / \mathrm{gr} \\
\text { (Mean(SD) }\end{array}$ & $79,23(46,88)$ & $71,91(44,37)$ & 0,04 \\
\hline $\begin{array}{l}\text { Total Cholesterol } \\
\mathrm{mg} / \mathrm{dl} \text { (Mean } \\
(\mathrm{SD}) \text { ) }\end{array}$ & $198,87(39,86)$ & $188,20(45,61)$ & $<0,001$ \\
\hline $\begin{array}{l}\text { LDL Cholesterol } \\
\mathrm{mg} / \mathrm{dl} \text { (Mean } \\
(\mathrm{SD}))\end{array}$ & $124,27(32,91)$ & $116,49(38,02)$ & $<0,001$ \\
\hline $\begin{array}{l}\mathrm{HDL} \text { Cholesterol } \\
\mathrm{mg} / \mathrm{dl} \text { (Mean(SD)) }\end{array}$ & $46(12,80)$ & $42,21(11,14)$ & $<0,001$ \\
\hline Triglycerides & Pras & more IAER ANI & $1 n$ \\
\hline
\end{tabular}

\section{Results:}

2200 patients were included: 30,45\% (670 patients) with type 2 diabetes, of which $90,72 \%$ had increased waist circumference by IDF criteria and $76,65 \%$ by Latin-American criteria.

In the 1st model,it was found: as independent factors associated with diabetes: Male gender OR = $1.30(95 \% \mathrm{Cl} 1,05-1,6, p=0,014)$ and increased waist circumference by IDF criteria OR $=1,44(95 \% \mathrm{CI}$ $1,02-2,04, p=0,038)$; as a protective factor HDL cholesterol OR = $0,97(95 \% \mathrm{Cl} 0,96-0.98, \mathrm{p}<0,001)$.

In the 2nd model, it was found: Male gender $\mathrm{OR}=1,31(95 \% \mathrm{Cl} 1,06-$ $1,61, p=0,01)$ and increased waist circumference by Latin-American criteria OR $=1,42(95 \% \mathrm{Cl} 1,12-1,79 ; p=0,003)$; as a protective factor HDL cholesterol OR = 0,97 (95\%Cl 0,96-0,98, p <0,001).

\begin{tabular}{|c|c|c|c|c|c|c|c|}
\hline \multicolumn{4}{|c|}{$\begin{array}{l}\text { Table No } 2 \text { Univariate analysis of factors } \\
\begin{array}{ll}\text { related to Type } 2 & \text { Diabetes }\end{array}\end{array}$} & \multicolumn{4}{|c|}{$\begin{array}{l}\text { Table No } 3 \text { Model } 1 \text { multivariate analysis of Waist } \\
\text { circumference increased by IDF criteria and factors related to } \\
\text { risk of Type } 2 \text { Diabetes }\end{array}$} \\
\hline Variables & OR & $\mathrm{Cl} 95 \%$ & $p$ & \begin{tabular}{|l|} 
Variable \\
\end{tabular} & OR & \begin{tabular}{l|l}
$\mathrm{Cl} 95 \%$ \\
\end{tabular} & \begin{tabular}{|l|}
$\mathrm{p}$ \\
\end{tabular} \\
\hline Age & 1,005 & $0,99-1,012$ & 0,18 & Age & 1,00 & $0,99-1,01$ & 0,407 \\
\hline Age stratum & & & & Male gender & 1,30 & $1,05-1,6$ & 0,014 \\
\hline 15 to 30 years & 1 & & & HDL Cholesterol & 0,97 & $0,96-0,98$ & $<0,001$ \\
\hline $\begin{array}{l}31 \text { to } 50 \text { years } \\
51 \text { to } 70 \text { years }\end{array}$ & $\begin{array}{l}0,84 \\
1,15\end{array}$ & $\begin{array}{l}0,38-1,83 \\
0,56-2,34\end{array}$ & $\begin{array}{l}0,66 \\
0,69\end{array}$ & Waist & & & \\
\hline$>70$ years & 1,12 & $0,55-2,29$ & \begin{tabular}{|l|}
0,74 \\
\end{tabular} & circumference & 144 & & \\
\hline Male gender & 1,41 & $1,17-1,69$ & $\begin{array}{c}<0,00 \\
1\end{array}$ & $\begin{array}{c}\text { increased by IDF } \\
\text { criteria }\end{array}$ & 1,44 & $1,02-2,04$ & 0,038 \\
\hline \multirow{2}{*}{$\begin{array}{c}\text { Waist } \\
\text { circumference } \\
\text { increased by } \\
\text { IDF criteria }\end{array}$} & \multirow{2}{*}{1,67} & \multirow{2}{*}{$1,24-2,24$} & \multirow{2}{*}{0,001} & \multirow{3}{*}{\multicolumn{4}{|c|}{ 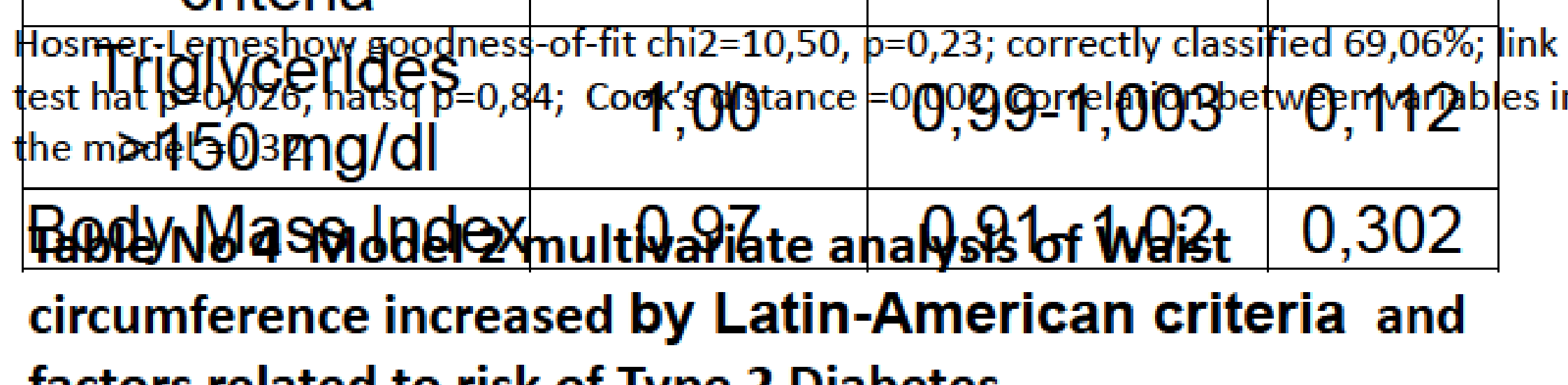 }} \\
\hline & & & & & & & \\
\hline \multirow{4}{*}{$\begin{array}{c}\text { Waist } \\
\text { circumference } \\
\text { by Latin- } \\
\text { American } \\
\text { criteria } \\
\end{array}$} & \multirow{4}{*}{1.55} & \multirow{4}{*}{$1,26-1,92$} & \multirow{4}{*}{$\begin{array}{c}<0,00 \\
1\end{array}$} & & & & \\
\hline & & & & Variable & OR & $\mathrm{Cl} 95 \%$ & $p$ \\
\hline & & & & Age & 1 & $0.99-1.013$ & 0.383 \\
\hline & & & & Male gender & 1.31 & $1.06-1.61$ & 0.01 \\
\hline Hypertension & 0,99 & $0,82-1,19$ & 0,94 & HDL Cholesterol & 0.97 & $0.96-0.98$ & $<0,001$ \\
\hline $\begin{array}{l}\text { Triglycerides } \\
>150 \mathrm{mg} / \mathrm{dl}\end{array}$ & 1,37 & $1,005-1,88$ & 0,046 & $\begin{array}{c}\text { Waist } \\
\text { circumference }\end{array}$ & & & \\
\hline Creatinine & 1,19 & $0,72-1,95$ & 0,49 & increased by & 1.42 & $1.12-1.79$ & 0.003 \\
\hline $\begin{array}{c}\text { LDL } \\
\text { Cholesterol }\end{array}$ & 0,99 & $\begin{array}{c}0,990- \\
0.996 \\
\end{array}$ & $\begin{array}{c}<0,00 \\
1\end{array}$ & $\begin{array}{l}\text { Latin-American } \\
\text { Hosmer-Leppifererpqoodness }\end{array}$ & &, 81 ; correctly classifiec & fied $69,01 \%$; link te \\
\hline $\begin{array}{c}\mathrm{HDL} \\
\text { Cholesterol } \\
\end{array}$ & 0,97 & $0,96-0,98$ & $\begin{array}{c}<0,00 \\
1\end{array}$ & 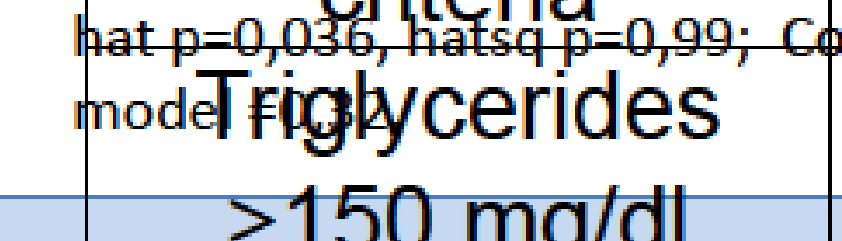 & & $\begin{array}{l}2 \text {; correlation betweer } \\
0.99-1.003\end{array}$ & $\begin{array}{l}3 \text { en variables in the } \\
0.256\end{array}$ \\
\hline OMgrlu & & $01-1,02$ & $\begin{array}{c}<0,00 \\
1\end{array}$ & \begin{tabular}{|l|}
$>150 \mathrm{mg} / \mathrm{dl}$ \\
Body Mass Index \\
\end{tabular} & 0.98 & $0.92-1.04$ & 0.592 \\
\hline
\end{tabular}

ACKNOWLEDGEMENTS

Writing assistance: Leonardo David Ospino Vera

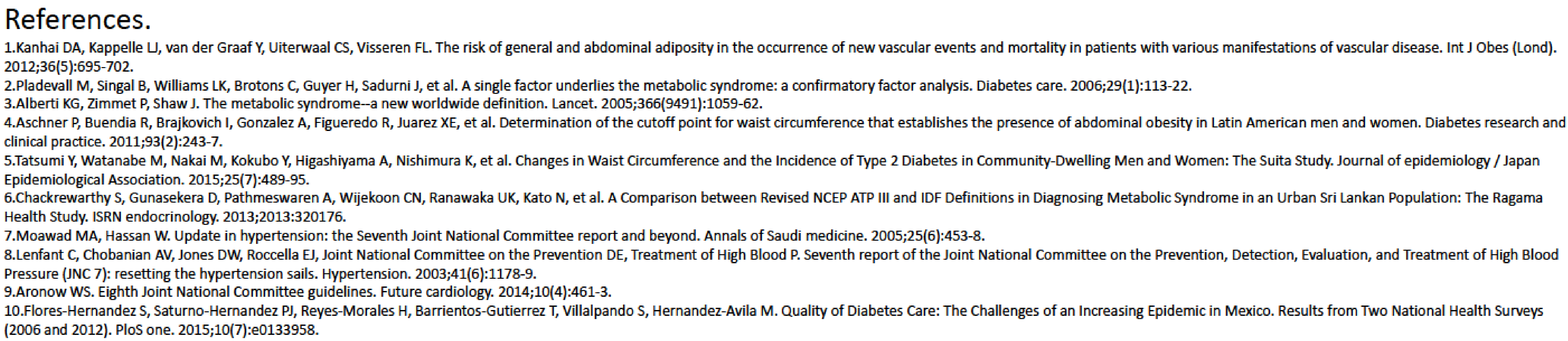

